\title{
Nonradial oscillations of slowly and differentially rotating compact stars
}

\author{
Adamantios Stavridis, ${ }^{1}$ Andrea Passamonti, ${ }^{1}$ and Kostas Kokkotas ${ }^{1}$ \\ ${ }^{1}$ Department of Physics, Aristotle University of Thessaloniki 54124, Greece
}

(Dated: October 30, 2018)

\begin{abstract}
The equations describing nonradial adiabatic oscillations of differentially rotating relativistic stars are derived in relativistic slow rotation approximation. The differentially rotating configuration is described by a perturbative version of the relativistic j-constant rotation law. Focusing on the oscillation properties of the stellar fluid, the adiabatic nonradial perturbations are studied in the Cowling approximation with a system of five partial differential equations. In these equations, differential rotation introduces new coupling terms between the perturbative quantites with respect to the uniformly rotating stars. In particular, we investigate the axisymmetric and barotropic oscillations and compare their spectral properties with those obtained in nonlinear hydrodynamical studies. The perturbative description of the differentially rotating background and the oscillation spectrum agree within a few percent with those of the nonlinear studies.

PACS numbers: 04.40.Dg, 95.30.Sf, 97.10.Sj
\end{abstract}

\section{INTRODUCTION}

Differential rotation can appear in many phases of the stellar evolution of a neutron star, such as in protoneutron stars [1, 2], in the massive remnant of binary neutron star mergers [3, 4], or as a results of stellar oscillations (r-modes) that may drive the star into differential rotation via nonliner effects [ $[\underline{5},[6,6$, , 8]. The differentially rotating phase can last at most some seconds depending on the dissipative mechanism that drive the star to uniform rotation, such as magnetic bracking [9, 10] and turbulent motion [11, 12].

However, this very short period can appear during the most violent phases of neutron star's life, as in the case of a core collapse or binary merging. This is exactly the time when we expect to get the strongest emission in gravitational waves. Since the ground based detectors reached sensitivities which allow the detection of gravitational wave signals from oscillating or unstable neutron stars the exact frequencies of the emitted waves are urgently needed. The study of linear pulsations and stability of differentially rotating stellar models started more than three decades ago both in Newtonian and relativistic gravity [13, 14, 15, 16]. The spectrum of differentially rotating stars in Newtonian theory has been studied in the frequency domain by [17].

Recently, the effects of differential rotation on the dynamical and secular instabilities of the $\mathrm{r}$ and $\mathrm{f}$-modes gained more attention. In Newtonian theory, the r-mode spectrum has been studied in [18, 19, 20], while the f-mode and the secular stability limits have been investigated in [21]. In stars that rotate with a high degree of differential rotation $\left(\Omega_{c} / \Omega_{s} \approx 10\right)$, an $m=2$ dynamical instability can appear even for considerably low rotation rates $\left(T /|W| \sim O\left(10^{-2}\right)\right)$ as suggested in [22, 23]. Note that $T$ is the rotational kinetic energy, $W$ the gravitational potential energy, $\Omega_{c}$ and $\Omega_{s}$ the angular velocity at the center and at the equatorial surface, respectively. In addition, an $m=1$ dynamical instability has been identified for high degrees of differential rotation and soft equations of state [24, 25]. More recently, it has been reported the discovery of $m=1$ and $m=2$ dynamical instability even for stiff equations of state [26]. Studies based on linear analysis [27, 28] suggest that low $T /|W|$ instabilities might be triggered when the corotation points of the unstable modes fall within the differentially rotating structure of the star. It is then evident that differentially rotating compact stars got a renewed interest and there are several recent fully general relativistic studies related to the issue. For example, slowly and differentially rotating magnetized neutron stars have been studied in [29, 30], the collapse and black hole formation of magnetized and differentially rotating neutron stars in [31] and the frequencies for nonlinear axisymmetric pulsations of differentially rotating stars in the Cowling and conformal flatness approximations in [32, 33].

As a further contribution to the direction of understanding stellar differential rotation we present here the equations describing a slowly and differentially rotating relativistic star. In our study we use a perturbative analysis and keep terms up to first order with respect to stellar rotation. In this first perturbative approach, we neglect all the spacetime perturbations i.e. we use the so called Cowling approximation since we want to focus on the behavior of the stellar fluid. The slow rotation approximation has been extensively used in the study of stellar perturbations both in Newtonian and in general relativistic approach [34, 35, 36, 37]. Here we study the spectrum of axisymmetric perturbations in order to test the accuracy of our approximation technique against published results by nonlinear numerical codes [32, 33]. The next steps will be to estimate the oscillation spectrum of the non-axisymmetric perturbations and furthermore to proceed in studying the low $T /|W|$ dynamical instability.

The structure of the paper is as follows. In section П we construct the differentially rotating stellar models, and 
test their accuracy against the equilibrium configurations obtained with non-linear codes. In section III, we derive the perturbation equations in the general case of non-barotropic and non-axisymmetric perturbations, which are numerically solved in section IV for the axisymmetric and barotropic case. In the section V , conclusions are drawn and the future applications are proposed. The appendix is organized in four sections. Section $₫$ is dedicated to the analytical expressions of the harmonic components of the j-constant rotation law, while all the coefficients of the perturbed conservation equations are given in section $\mathrm{B}$. The operators that couple perturbations with different harmonic indices are introduced in section $\mathrm{C}$. Finally, in the last section $\mathrm{D}$ we discuss the j-constant rotation law in isotropic coordinates.

Throughout the paper we use geometrical units $c=G=1$. We use a prime (' $)$ to denote derivatives with respect to the radial coordinate $r$ and the overdot $\left({ }^{*}\right)$ for derivatives with respect to the time coordinate $t$.

\section{EQUILIBRIUM CONFIGURATION}

The axially symmetric spacetime of a slowly and differentially rotating star can be described by the following line element:

$$
d s^{2}=-e^{2 \nu} d t^{2}+e^{2 \lambda} d r^{2}-2 \omega r^{2} \sin ^{2} \theta d t d \phi+r^{2}\left(d \theta^{2}+\sin ^{2} \theta d \phi^{2}\right),
$$

where $\nu, \lambda$ are scalar fields that depend on the radial coordinate $r$. The metric function $\omega$, which describes the dragging of the reference frames due to the stellar rotation, is a function of the both $r$ and $\theta$ coordinates. In this paper, we assume that the stellar interior is described by the perfect fluid energy-momentum tensor:

$$
T^{\alpha \beta}=(\epsilon+p) u^{\alpha} u^{\beta}+p g^{\alpha \beta},
$$

where $\epsilon$ and $p$ denote the total energy density and the pressure respectively, and $u^{\alpha}$ is the fluid velocity

$$
u^{\alpha}=\left(e^{-\nu}, 0,0, \Omega e^{-\nu}\right),
$$

where $\Omega=\Omega(r, \theta)$ is the angular velocity of the star as measured by an observer at infinity.

By providing an equation of state $(\mathrm{EoS}) p=p(\epsilon)$, a rotating equilibrium configuration can be determined by solving the Tolman-Oppenheimer-Volkoff (TOV) equations together with the following equation for the metric function $\omega$ :

$$
\omega^{\prime \prime}-\left[4 \pi(\epsilon+p) r e^{2 \lambda}-\frac{4}{r}\right] \omega^{\prime}-\left[16 \pi(\epsilon+p)+\frac{l(l+1)-2}{r^{2}}\right] e^{2 \lambda} \omega=-16 \pi(\epsilon+p) e^{2 \lambda} \Omega .
$$

\section{A. Rotation Law}

In general the differential profile of the angular velocity $\Omega$ in not known. Still approximate analytic differential rotating laws have been adopted and used both for Newtonian and General Relativistic configurations (see [38] and reference therein). Among these laws, the j-constant rotation law [39, 40] guarantees Rayleigh's stability against axisymmetric perturbations for inviscid fluids, and seems to describe quite well not only typically differentially rotating stars but even the remnant rotational configurations that arise from the merging of hypermassive binary systems [4].

The relativistic j-constant rotation law can be perturbatively expanded as in [41]:

$$
\Omega(r, \theta)=\frac{A^{2} \Omega_{c}+e^{-2 \nu} \omega(r, \theta) r^{2} \sin ^{2} \theta}{A^{2}+e^{-2 \nu} r^{2} \sin ^{2} \theta},
$$

where $\Omega_{c}$ denotes the angular velocity at the rotation axis, while $A$ is the parameter that describes the degree of differential rotation of the star. For high values of $A$ (e.g. $A \sim 500 \mathrm{~km})$, the j-constant rotation law (5) tends to a uniformly rotating configuration, where $\Omega \rightarrow \Omega_{c}$.

In the slow rotation approximation, rotation is treated as a perturbation of a spherically symmetric background spacetime. Therefore, the tensor harmonic expansion enables us to separate the angular dependence of any perturbative fields from the temporal and radial parts. In this way the metric function $\omega$ and the angular velocity $\Omega$ of the fluid can then be written as follows:

$$
\begin{aligned}
& \omega(r, \theta)=-\sum_{l} \omega_{l}(r) \frac{1}{\sin \theta} \frac{\partial P_{l}}{\partial \theta} \\
& \Omega(r, \theta)=-\sum_{l} \Omega_{l}(r) \frac{1}{\sin \theta} \frac{\partial P_{l}}{\partial \theta}
\end{aligned}
$$


where $P_{l}(\cos \theta)$ are the Legendre's polynomials. The $\Omega_{l}$ and $\omega_{l}$ components in equations (6)-(7) are then determined via the orthogonality relations of the form,

$$
f_{l}(r)=-\frac{1}{4 \pi} \frac{2 l+1}{l(l+1)} \int_{S^{2}} d \tilde{\Omega} f(r, \theta) \sin \theta \frac{\partial P_{l}}{\partial \theta},
$$

where $f=f(r, \theta)$ is a scalar field. In equation (8), $d \tilde{\Omega}$ denotes the volume element of the 2 -sphere $S^{2}$. It is worth mentioning that for a uniformly rotating star, the only non vanishing components of equations (6)-(7) are the ones for $l=1$ [42]. In this case, $\omega$ depend only on the radial coordinate $r$ and $\Omega$ is of course constant. For differentially rotating stars, which are described by the j-constant rotation law, the components $\Omega_{l}$ can be determined by direct substitution of equation (5) in (8). Then the resulting expression can be split in two parts:

$$
\Omega_{l}=\mathcal{I}_{l}^{N}+\mathcal{I}_{l}^{R},
$$

where $\mathcal{I}_{l}^{N}$ is the "nearly Newtonian" part and $\mathcal{I}_{l}^{R}$ is the "relativistic" correction. The definition of these two terms is given by the following expressions:

$$
\begin{aligned}
\mathcal{I}_{l}^{N} & \equiv-\frac{2 l+1}{l(l+1)} \frac{\Omega_{c}}{4 \pi} \int_{S^{2}} d \tilde{\Omega} \frac{A^{2} \sin \theta}{A^{2}+e^{-2 \nu} r^{2} \sin ^{2} \theta} \frac{\partial P_{l}}{\partial \theta}, \\
\mathcal{I}_{l}^{R} & \equiv-\frac{2 l+1}{l(l+1)} \frac{e^{-2 \nu} r^{2}}{4 \pi} \int_{S^{2}} d \tilde{\Omega} \frac{\sin ^{3} \theta \omega(r, \theta)}{A^{2}+e^{-2 \nu} r^{2} \sin ^{2} \theta} \frac{\partial P_{l}}{\partial \theta} .
\end{aligned}
$$

The parity of the j-constant rotation law implies that the non-vanishing components in (10)-(11) are the ones with odd $l$. The integral $\mathcal{I}_{l}^{N}$ for $l=1$ and $l=3$ has been already calculated in [41], and is analytically written in appendix [A. Moreover, the integration of the "relativistic" term $\mathcal{I}_{l}^{R}$ requires an additional expansion of the metric variable $\omega$. Therefore, by inserting equation (6) into (11) one gets

$$
\mathcal{I}_{l}^{R}=\frac{2 l+1}{l(l+1)} \frac{e^{-2 \nu} r^{2}}{4 \pi} \sum_{l^{\prime}} \omega_{l^{\prime}}(r) \mathcal{J}_{l}^{l^{\prime}}
$$

where $\mathcal{J}_{l}^{l^{\prime}}$ are given by the following integral relation

$$
\mathcal{J}_{l}^{l^{\prime}} \equiv \int_{S^{2}} d \tilde{\Omega} \frac{\sin ^{2} \theta}{A^{2}+e^{-2 \nu} r^{2} \sin ^{2} \theta} \frac{\partial P_{l}}{\partial \theta} \frac{\partial P_{l^{\prime}}}{\partial \theta} .
$$

The detailed forms of $\mathcal{J}_{1}^{1}, \mathcal{J}_{1}^{3}$ and $\mathcal{J}_{3}^{3}$ are shown in appendix A, Our choice to retain only the $l=1$ and $l=3$ terms for the expression (13) will be justified later in section [V] were we show that the frequencies of the nonradial oscillations that we calculate using this approach are in very good agreement with the ones derived using the full nonlinear evolution of the fluid background [32, 33].

\section{B. Frame dragging in differential rotation}

The various components $\omega_{l}$ of the series expansion (6) which provides $\omega$ will be determined from the solution of equation (4) for the frame dragging. In practice, one substitutes equations (6) and (7) into equation (4), and collects the terms of the series with the same index $l$. However, the term $\mathcal{I}_{l}^{R}$ describing the relativistic correction, equation (12), introduces a coupling between the different harmonics, which is due to the presence of metric function $\omega$ in equation (5). As a result the terms $\omega_{l}$ will be determined as solutions of a system of coupled ordinary differential equations. In order to simplify the presentation of this system of differential equations we will write it schematically as:

$$
\mathcal{L}(\omega)=\mathcal{S}(\Omega)
$$

where $\mathcal{L}(\cdot)$ represents the linear differential operator of the left hand side of equation (4), while $\mathcal{S}(\cdot)$ denotes the linear operator of the source. When equation (14) is expanded into vector harmonics, it reduces to the following set of equations

$$
\mathcal{L}\left(\omega_{l}\right)=\mathcal{S}\left(\mathcal{I}_{l}^{N}\right)+\frac{2 l+1}{l(l+1)} \frac{e^{-2 \nu} r^{2}}{4 \pi} \sum_{l^{\prime}} \mathcal{S}\left(\omega_{l^{\prime}} \mathcal{J}_{l}{ }^{l^{\prime}}\right),
$$

which applies to any value of $l$. The first two non-vanishing terms $\omega_{1}$ and $\omega_{3}$ obey the following coupled system of equations: 


\begin{tabular}{cccc}
\hline \hline Model & $T_{c}(\mathrm{~ms})$ & $\Omega_{c}\left(\times 10^{-2}\right) \mathrm{km}^{-1}$ & $\Omega_{e}\left(\times 10^{-2}\right) \mathrm{km}^{-1}$ \\
\hline B1 & 1.719 & 1.218 & 0.435 \\
B2 & 1.204 & 1.740 & 0.621 \\
B3 & 0.970 & 2.160 & 0.771 \\
B6 & 0.657 & 3.189 & 1.139 \\
B9 & 0.496 & 4.219 & 1.507 \\
\hline \hline
\end{tabular}

TABLE I: Properties of the background models used in the paper. The quantities $T_{c}$ and $\Omega_{c}$ are respectively the period and the angular velocity at the rotational axis, while $\Omega_{e}$ represents the angular velocity at the equator. All stellar models have mass $M=1.40 M_{\odot}$ and radius $R=14.151 \mathrm{~km}$.

$$
\begin{aligned}
\mathcal{L}\left(\omega_{1}\right)-\frac{3}{8 \pi} r^{2} e^{-2 \nu} \mathcal{S}\left(\omega_{1} \mathcal{J}_{1}^{1}\right) & =\mathcal{S}\left(\mathcal{I}_{1}^{N}\right)+\frac{3}{8 \pi} r^{2} e^{-2 \nu} \mathcal{S}\left(\omega_{3} \mathcal{J}_{1}^{3}\right) \\
\mathcal{L}\left(\omega_{3}\right)-\frac{7}{48 \pi} r^{2} e^{-2 \nu} \mathcal{S}\left(\omega_{3} \mathcal{J}_{3}^{3}\right) & =\mathcal{S}\left(\mathcal{I}_{3}^{N}\right)+\frac{7}{48 \pi} r^{2} e^{-2 \nu} \mathcal{S}\left(\omega_{1} \mathcal{J}_{3}^{1}\right)
\end{aligned}
$$

which together with a set of appropriate boundary conditions at the stellar center and at infinity form a boundary value problem. Actually, the demand for regularity of the solutions at the stellar center and their decaying asymptotic behavior suggest the following approximate relations [43]:

$$
\begin{aligned}
& \omega_{l} \sim r^{l-1}, \quad \text { for } r \rightarrow 0, \\
& \omega_{l} \sim r^{-l-2}, \quad \text { for } r \rightarrow \infty .
\end{aligned}
$$

The numerical method used for the solution of the above system of equations is described in the next paragraphs.

\section{Stellar Models}

In order to test the accuracy of the perturbative treatment of stellar differential rotation, which has been introduced in this paper, we will compare our results with those derived from the numerical solution of the nonlinear Einstein equations. Thus we will study a set of stellar models, the so-called B models, which have been already adopted in [32, 33]. These models describe barotropic and differentially rotating stars, where the rotating pattern is described by the $\mathrm{j}$-constant rotation law. For simplicity, a relativistic barotropic EoS of the form

$$
\begin{aligned}
& p=K \rho^{\Gamma}, \\
& \epsilon=\rho+\frac{p}{\Gamma-1},
\end{aligned}
$$

has been adopted, where $\rho$ is the rest mass density and $K$ and $\Gamma$ the polytropic parameters.

For a given EoS, an equilibrium configuration depends on three parameters: the central density $\rho_{c}$, the stellar spin at the rotation axis $\Omega_{c}$ and the parameter $A$ describing the degree of differential rotation. For the $\mathrm{B}$ models the polytropic parameters get the values $K=217.858 \mathrm{~km}^{2}$ and $\Gamma=2$ while the central rest mass density is fixed to the value $\rho_{\mathrm{c}}=5.87 \times 10^{-4} \mathrm{~km}^{-2}$. The solution of the TOV equations provide a stellar model with mass $M=1.40 M_{\odot}$ and radius $R=14.151 \mathrm{~km}$. The angular velocity at the rotation axis, $\Omega_{c}$, for some of the B1-B9 models is listed in table 1 . In order to compare our results with those of nonlinear studies special care has been taken for the interpretation of the parameter $A$, due to the different coordinate systems that are commonly used in non-linear studies and our perturbative approximation. This issue is explained in detail in appendix $\mathrm{D}$, where we show that $A$ should be set to the value of the isotropic stellar radius.

As we mentioned earlier the two components $\omega_{1}$ and $\omega_{3}$ of the metric function $\omega$ will be the solutions of the boundary value problem described by the two differential equations (16)-(17) together with the boundary conditions (18)-(19). By discretizing equations (16)-(17) using a second order scheme one can construct a tridiagonal linear system. This system can be solved using a standard LU decomposition where the boundary conditions (18) and (19) are appropriately implemented. The presence of coupling terms on the right hand side of the equations calls for an iterative treatment. As a first step a trial solution $\omega_{l}^{\text {tr }}$ of (16)-(17) can be determined by neglecting the coupling source terms, i.e. $\mathcal{J}_{1}^{3}=\mathcal{J}_{3}^{1}=0$. This trial solution is then substituted only into the source terms and the system of 


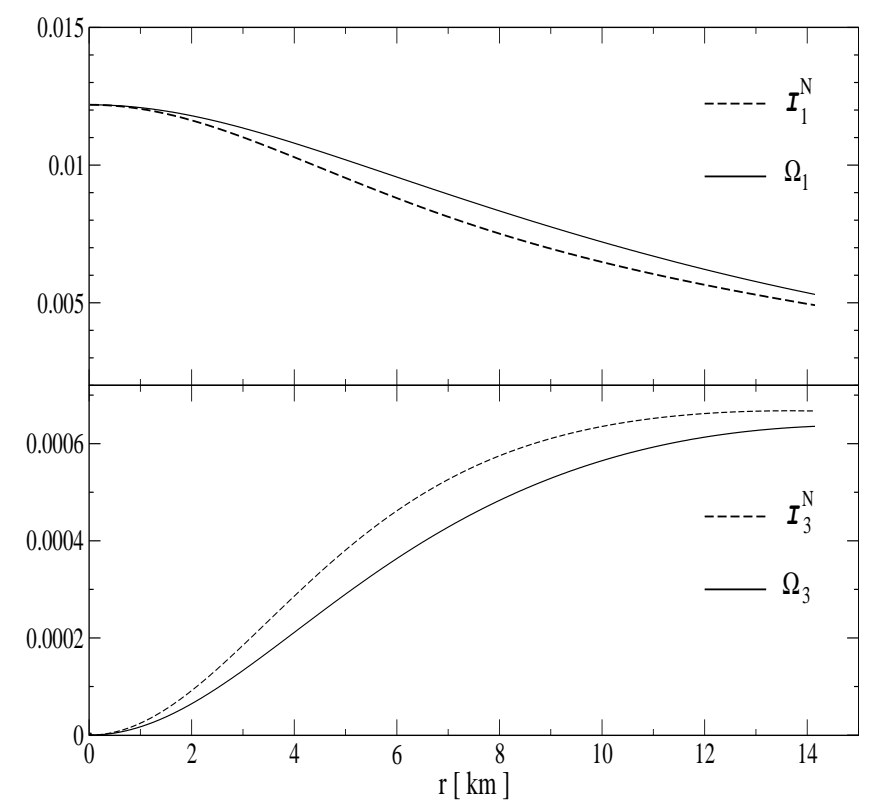

FIG. 1: The harmonic components $\Omega_{1}$ (upper panel) and $\Omega_{3}$ (lower panel) of the angular velocity $\Omega$ of the B1 stellar model are plotted, where $\Omega_{c}=1.2210^{-2} \mathrm{~km}^{-1}$ and $A=12 \mathrm{~km}$. The dashed lines display the contribution of the "nearly Newtonian term" given by equation (10).

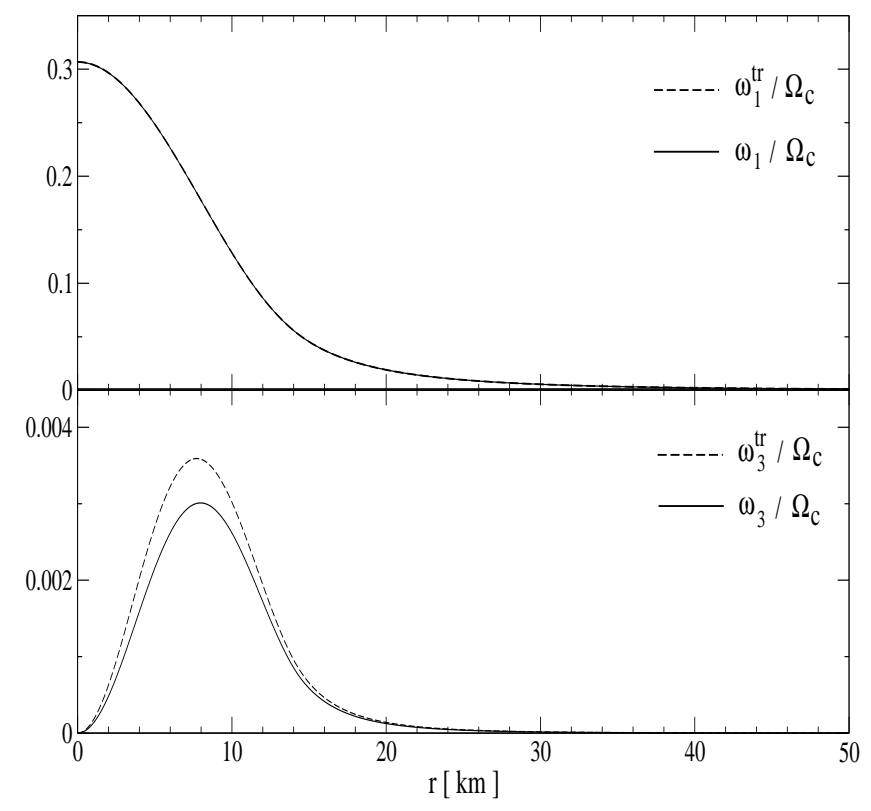

FIG. 2: The harmonic components $\omega_{1}$ (upper panel) and $\omega_{3}$ (lower panel) of the angular velocity $\omega$ of the B1 stellar model are plotted. The dashed lines denote the trial solutions of equation (16), which have been determined without the coupling terms $\mathcal{J}_{3}^{1}$ and $\mathcal{J}_{1}^{3}$. The solid lines are instead the final solutions.

equations (16)-(17) is solved. This procedure is repeated until the solution converges. In practice, only three or four iterations are needed.

In figures 1, 4 we show the behavior of the angular velocity $\Omega$ and the metric function $\omega$ for the slowly rotating model B1, see table [. In figure 1 it is noticeable the small contribution of the "relativistic corrections" $\mathcal{I}_{R}^{l}$ in the estimation the star's angular velocity. The two components of the metric function i.e. $\omega_{1}$ and $\omega_{3}$ are plotted in figure 2 together with initial trial solutions $\omega_{l}^{\text {tr }}$. It is worth noticing an imperceptible correction to the initial trial solution for $l=1$, 


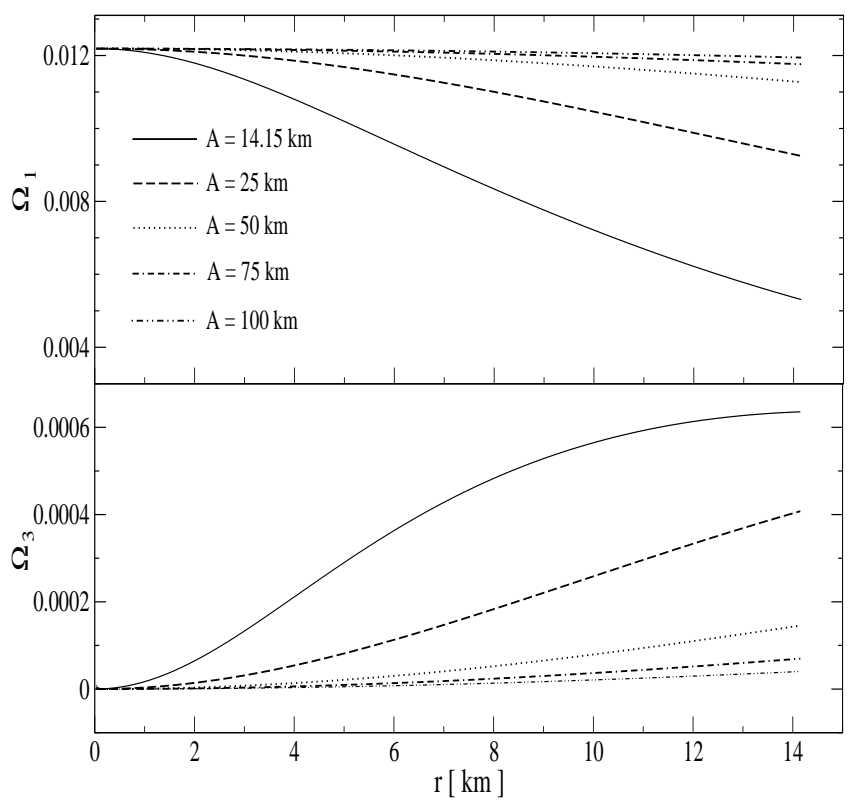

FIG. 3: The dependence of the two components of the angular velocity $\Omega$ on the differential parameter $A$ is shown in this figure for the stellar model B1.

and the small value of $\omega_{3}$ with respect to $\omega_{1}$, which suggests that terms of the perturbative series (6)-(7) with higher $l$ will not affect the results. The dependence of $\Omega_{1}$ and $\Omega_{3}$ on the the differential rotation parameter $A$ is illustrated in figure 3. There one can notice that for increasing values of $A$ the $\Omega_{1}$ reaches its uniform rotation value $\Omega_{c}$, while $\Omega_{3}$ decreases proportionally as expected.

In order to estimate the accuracy of our approximate solutions for $\Omega$ and $\omega$, we have done a comparison with the profiles derived by the nonlinear numerical code RNS [44]. The results from RNS and the perturbative solutions for the B1-model are compared in figure 4 on the equatorial plane. The maximum deviation between the two estimations is around $r=6 \mathrm{~km}$ where the relative error is about $5.5 \%$. The accuracy could be further improved by taking into account the $l=5$ contributions. However, the nonradial perturbation equations would become considerably more complicated due to the presence of extra coupling terms. As we show in the next section the approximation used here allows the estimation of the nonradial oscillations frequencies with an error smaller than 10 percent even for very rapidly rotating models, see table III.

\section{PERTURBATION EQUATIONS}

In this section, we derive the perturbation equations which describe the nonradial oscillations of a slowly and differentially rotating star. The rotation, as described in the previous section, is treated perturbatively up to the first order in $\Omega$ [45]. In this work we focus on the spectral properties of the fluid modes, therefore, we neglect all metric perturbations, and we use the so-called "Cowling approximation". For slowly rotating stars, this method enables us to determine the frequencies of nonradial pulsations with an accuracy to better than $15 \%$ with respect to the non approximated approach. In addition, the accuracy improves for modes with higher harmonic index $l$ and mode order $n$ [46].

We will consider adiabatic oscillations, where the pressure and density perturbations are related by the following expression:

$$
\delta p=\frac{\Gamma_{1} p}{p+\epsilon} \delta \epsilon+p^{\prime} \xi^{r}\left(\frac{\Gamma_{1}}{\Gamma}-1\right)
$$

where $\xi^{r}$ is the radial component of the fluid displacement vector $\xi^{\mu}$ and $\Gamma$ and $\Gamma_{1}$ represent the adiabatic index of the background and perturbed configurations respectively. The adiabatic index $\Gamma$ is defined by the following relation:

$$
\Gamma=\frac{p+\epsilon}{\epsilon} \frac{d p}{d \epsilon}
$$



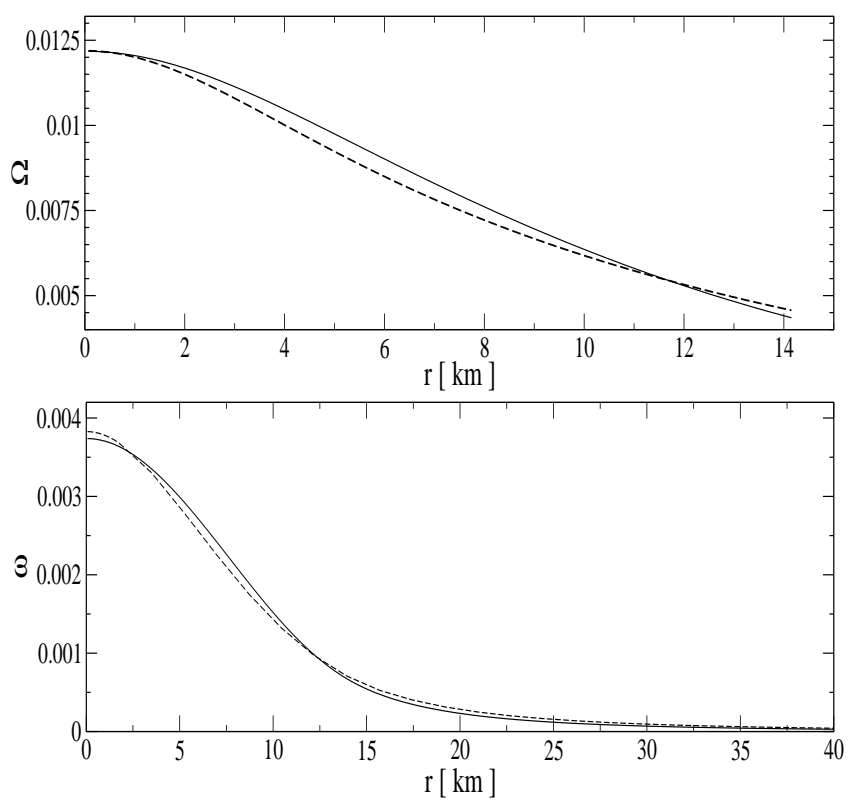

FIG. 4: The profiles of $\Omega$ and $\omega$ derived from the perturbative treatment and the nonlinear RNS code compared in this figure. The solid lines represent the perturbative solutions and dashed lines the RNS quantities. In the upper panel the estimations of the angular velocity $\Omega$ are plotted, while in the lower panel the variable $\omega$ is compared to the shift component $-\beta^{\phi}$, which have been determined with the RNS code.

and sound speed by

$$
c_{s}^{2}=\frac{\Gamma_{1}}{\Gamma} \frac{d p}{d \epsilon} .
$$

In Cowling approximation, the displacement vector $\xi^{\mu}$ is related to the 4 -velocity perturbation by

$$
\delta u_{\mu}=g_{\mu \nu} u^{\lambda} \frac{\partial \xi^{\nu}}{\partial x^{\lambda}} .
$$

The $r$ component of this equation provides an evolution equation for $\xi^{r}$ :

$$
\left(\partial_{t}+\Omega \partial_{\phi}\right) \xi^{r}=e^{-2 \lambda+\nu} \delta u_{r} .
$$

The three scalar quantities describing the perturbations of the total energy density, pressure and the $r$ component of the Lagrangian displacement can be expanded in spherical harmonics,

$$
\begin{aligned}
\delta \epsilon & =\delta \epsilon_{l m} Y_{l m} \\
\delta p & =\delta p_{l m} Y_{l m} \\
\xi^{r} & =\left[\nu^{\prime}\left(1-\frac{\Gamma_{1}}{\Gamma}\right)\right]^{-1} \xi^{l m} Y_{l m} .
\end{aligned}
$$

The perturbation equations can be considerably simplified if one uses a new function $H$, which is related to the energy density and pressure perturbations by the following relations

$$
\begin{aligned}
\delta p_{l m} & =H_{l m}(p+\epsilon), \\
\delta \epsilon_{l m} & =\frac{(p+\epsilon)^{2}}{p \Gamma_{1}}\left(H_{l m}-\xi_{l m}\right) .
\end{aligned}
$$

For barotropic fluids, $H$ becomes the perturbation of the relativistic enthalpy [47]. Velocity perturbations are also expanded into vector harmonic expansion and have the form: 


$$
\begin{aligned}
\delta u_{r} & =-e^{\nu} \sum_{l m} u_{1, l m} Y_{l m}, \\
\delta u_{\theta} & =-e^{\nu} \sum_{l m}\left(u_{2, l m} \frac{\partial Y_{l m}}{\partial \theta}-u_{3, l m} \frac{\partial Y_{l m}}{\partial \phi} \frac{1}{\sin \theta}\right), \\
\delta u_{\phi} & =-e^{\nu} \sum_{l m}\left(u_{2, l m} \frac{\partial Y_{l m}}{\partial \phi}+u_{3, l m} \sin \theta \frac{\partial Y_{l m}}{\partial \theta}\right), \\
\delta u_{t} & =-\Omega(r, \theta) \delta u_{\phi}=-e^{\nu} \sum_{l^{\prime} m^{\prime}} \sum_{l m}\left(u_{2, l m} \frac{\partial Y_{l m}}{\partial \phi}+u_{3, l m} \sin \theta \frac{\partial Y_{l m}}{\partial \theta}\right) \Omega_{l^{\prime} m^{\prime}}(r) \frac{1}{\sin \theta} \frac{\partial P_{l^{\prime}}}{\partial \theta},
\end{aligned}
$$

notice that in the last expression equation (7) has been used.

Due to the parity of the tensor harmonics, the nonradial perturbations can be divided in two classes, the axial (oddparity) and polar (even-parity) perturbations. The polar sector can be described by four perturbation functions: the perturbation of the relativistic enthalpy $H(r, t)$, the two velocity perturbations $u_{1}(t, r), u_{2}(t, r)$, and the $r$ component of the displacement vector, $\xi^{r}(r, t)$. On the other hand, in the Cowling approximation, axial perturbations will be described only by the velocity perturbation function $u_{3}(t, r)$. Using the perturbed energy-momentum conservation equations $\delta\left(T_{\alpha \beta} ; \beta\right)=0$ one can derive a system of coupled differential equations for $H_{l m}$ and the $u_{i, l m}$ which together with equation (26) form a complete linear system of evolution equations for the five unknown perturbation functions. The four components of the perturbed energy-momentum conservation equations get the form:

$$
\begin{aligned}
& A_{l m}^{(t)} Y_{l m}+\left[B_{1, l m}^{(t)}+B_{2, l m}^{(t)} \sin ^{2} \theta\right] \frac{\partial Y_{l m}}{\partial \phi}=0 \\
& {[r] \quad A_{l m}^{(r)} Y_{l m}+\left[B_{1, l m}^{(r)}+B_{2, l m}^{(r)} \sin ^{2} \theta\right] \frac{\partial Y_{l m}}{\partial \phi}+\left[C_{1, l m}^{(r)}+C_{2, l m}^{(r)} \sin ^{2} \theta\right] \sin \theta \frac{\partial Y_{l m}}{\partial \theta}=0 } \\
& {[\theta] \quad\left[a_{1, l m}+a_{2, l m} \cos \theta+a_{3, l m} \sin ^{2} \theta+a_{4, l m}^{(\theta)} \cos \theta \sin ^{2} \theta\right] \frac{\partial Y_{l m}}{\partial \theta} } \\
&-\quad\left[b_{1, l m}+b_{2, l m} \cos \theta+b_{3, l m} \sin ^{2} \theta+b_{4, l m}^{(\theta)} \cos \theta \sin ^{2} \theta\right] \frac{1}{\sin \theta} \frac{\partial Y_{l m}}{\partial \phi}=0 \\
& {[\phi] } \\
& \quad\left[b_{1, l m}+b_{2, l m} \cos \theta+b_{3, l m} \sin ^{2} \theta+b_{4, l m}^{(\phi)} \cos \theta \sin ^{2} \theta\right] \frac{\partial Y_{l m}}{\partial \theta} \\
&+\quad\left[a_{1, l m}+a_{2, l m} \cos \theta+a_{3, l m} \sin ^{2} \theta+a_{4, l m}^{(\phi)} \cos \theta \sin ^{2} \theta\right] \frac{1}{\sin \theta} \frac{\partial Y_{l m}}{\partial \phi}+\left[c_{1, l m}^{(\phi)}+c_{2, l m}^{(\phi)} \sin ^{2} \theta\right] \sin \theta Y_{l m}=0,
\end{aligned}
$$

where all the coefficients $A_{l m}, B_{l m}, C_{l m}, a_{i, l m}, b_{i, l m}$ and $c_{i, l m}$ are analytically written in appendix B. Notice that in equations (26) and (36)-(39), the background variables $\omega$ and $\Omega$ are expanded up to the harmonic index $l=3$. The angular dependence of the above system of equations is removed by performing angular integrations. As a result, we obtain the following system of coupled evolution equations for $H_{l m}, u_{1, l m} u_{2, l m}, u_{3, l m}$ and $\xi_{l m}^{r}$ : 


\begin{tabular}{ccc}
\hline \hline$l_{\max }$ & F (kHz) & $H_{1}(\mathrm{kHz})$ \\
\hline 0 & 2.687 & 4.551 \\
1 & 2.710 & 4.571 \\
2 & 2.712 & 4.575 \\
3 & 2.712 & 4.575 \\
\hline \hline
\end{tabular}

TABLE II: Oscillation frequencies of the quasi-radial $\mathrm{F}$ and $H_{1}$ modes for the $\mathrm{B} 1$ stelar model. With $l_{\text {max }}$ we denote the maximum harmonic index that has been used in the estimation. It is apparent that there is a fast convergence towards the exact value for relative small values of $l_{\max }$.

$$
\begin{aligned}
A_{l m}^{(t)} & +\mathrm{i} m\left[B_{1, l m}^{(t)}+\mathcal{L}_{1}^{ \pm 2} B_{2, l m}^{(t)}\right]=0 \\
A_{l m}^{(r)} & +\mathrm{i} m\left[B_{1, l m}^{(r)}+\mathcal{L}_{1}^{ \pm 2} B_{2, l m}^{(r)}\right]+\left[\mathcal{L}_{1}^{ \pm 1} C_{1, l m}^{(r)}+\mathcal{L}_{1}^{ \pm 3} C_{2, l m}^{(r)}\right]=0, \\
\Lambda a_{1, l m} & -\mathrm{i} m\left[b_{2, l m}-2 \mathcal{L}_{4}^{ \pm 1} b_{3, l m}+\mathcal{L}_{2}^{ \pm 2} b_{4, l m}^{(\theta)}+\mathcal{L}_{3}^{ \pm 2} b_{4, l m}^{(\phi)}+c_{1, l m}^{(\phi)}+\mathcal{L}_{1}^{ \pm 2} c_{2, l m}^{(\phi)}\right] \\
& +\mathcal{L}_{3}^{ \pm 1} a_{2, l m}+\mathcal{L}_{4}^{ \pm 2} a_{3, l m}+\mathcal{L}_{2}^{ \pm 3} a_{4, l m}^{(\theta)}+m^{2} \mathcal{L}_{4}^{ \pm 1} a_{4, l m}^{(\phi)}=0 \\
\Lambda b_{1, l m} & +\mathrm{i} m\left[a_{2, l m}-2 \mathcal{L}_{4}^{ \pm 1} a_{3, l m}+\mathcal{L}_{3}^{ \pm 2} a_{4, l m}^{(\theta)}+\mathcal{L}_{2}^{ \pm 2} a_{4, l m}^{(\phi)}\right] \\
& +\mathcal{L}_{3}^{ \pm 1} b_{2, l m}+\mathcal{L}_{4}^{ \pm 2} b_{3, l m}+\mathcal{L}_{2}^{ \pm 3} b_{4, l m}^{(\phi)}+m^{2} \mathcal{L}_{4}^{ \pm 1} b_{4, l m}^{(\theta)}+\mathcal{L}_{2}^{ \pm 1} c_{1, l m}^{(\phi)}+\mathcal{L}_{3}^{ \pm 3} c_{2, l m}^{(\phi)}=0 . \\
\dot{\xi}_{l m} & +\mathrm{i} m\left(\Omega_{10}+6 \Omega_{30}\right) \xi_{l m}=e^{2 \nu-2 \lambda}\left(1-\frac{\Gamma_{1}}{\Gamma}\right) \nu^{\prime} u_{1, l m}+\frac{15}{2} \Omega_{30} \mathcal{L}_{1}^{ \pm 2} \xi_{l m} .
\end{aligned}
$$

where $\Lambda=l(l+1)$. The operators $\mathcal{L}_{i}^{ \pm j}$ couple perturbations with different harmonic indeces $l$ and they are defined in appendix C. The final equations can be written in a more compact form as,

$$
\begin{aligned}
& P_{l m}+\mathrm{i} m\left(P_{l m}+\tilde{A}_{l \pm 1, m}+\tilde{P}_{l \pm 2, m}\right)+A_{l \pm 1, m}+\tilde{A}_{l \pm 3, m}=0, \\
& A_{l m}+\mathrm{i} m\left(A_{l m}+\tilde{P}_{l \pm 1, m}+\tilde{A}_{l \pm 2, m}\right)+P_{l \pm 1, m}+\tilde{P}_{l \pm 3, m}=0,
\end{aligned}
$$

where $P_{l m}$ and $A_{l m}$ represent polar and axial perturbation functions respectively. The tilded quantities denote the extra coupling terms introduced by the differentially rotating background. It is worth noticing that apart from the $l \pm 1$ couplings existing in the case of uniform rotation we have extra couplings $l \pm 2$ and $l \pm 3$ introduced by the differential rotation.

According to the classification of [48] the above system of infinite coupled equations consists of two decoupled sub-systems among which there is no energy exchange. The first one is the so called "axial-led" system and consists of the polar radial $l=0$ perturbations that couple with the axial non-radial $l=1$, the polar non-radial $l=2$ and so on. The second system is the so called "polar-led" and consists of the polar dipole $l=1$ perturbations that couple with the axial non-radial $l=2$, the polar non-radial $l=3$ and so on. Since the two sub-systems are decoupled in order to excite both we have to give independent appropriate initial data for time evolutions. In our study, for both polar and axial functions with $l=2$ we assumed a generic gaussian pulse an an initial perturbation. The boundary conditions on the stellar surface are set by the vanishing of the Langrangian perturbation of the pressure $\Delta P=0$.

\section{NUMERICAL RESULTS}

The perturbation equations derived in the previous sections have been studied numerically in order to compare the accuracy of the present approach against published results by nonlinear evolution codes [32]. More specifically we study the spectral properties of axisymmetric $(m=0)$ nonradial oscillations of a slowly and differentially rotating star, where the fluid is described by a barotropic $\operatorname{EoS}\left(\Gamma_{1}=\Gamma\right)$. An extensive study of non-axisymmetric perturbations 
of differentially rotating relativistic stars for a wide range of EoS will be the subject of a follow up paper [49]. In the Cowling approximation, for barotropic and axisymmetric $(m=0)$ perturbations the system of evolution equations (40)-(44) simplifies considerably and reduces to the following four coupled equations:

$$
\begin{aligned}
\dot{H}_{l} & =\left\{\left[\left(\frac{2}{r}-\lambda^{\prime}+2 \nu^{\prime}\right) c_{s}^{2}-\nu^{\prime}\right] u_{1, l}+c_{s}^{2} u_{1, l}^{\prime}\right\} e^{2(\nu-\lambda)}-c_{s}^{2} \Lambda \frac{e^{2 \nu}}{r^{2}} u_{2, l}, \\
\dot{u}_{1, l} & =H_{l}^{\prime}+\left\{\left[2\left(\frac{1}{r}-\nu^{\prime}\right)\left(\varpi_{1}+6 \varpi_{3}\right)-\omega_{1}^{\prime}-6 \omega_{3}^{\prime}\right] \mathcal{L}_{1}^{ \pm 1}-\frac{15}{2}\left[2\left(\frac{1}{r}-\nu^{\prime}\right) \varpi_{3}-\omega_{3}^{\prime}\right] \mathcal{L}_{1}^{ \pm 3}\right\} u_{3, l}, \\
\dot{u}_{2, l} & =H_{l}+\frac{1}{\Lambda}\left\{2\left(\varpi_{1}+6 \varpi_{3}\right) \mathcal{L}_{3}^{ \pm 1}-15\left(\Omega_{3}-2 \omega_{3}\right) \mathcal{L}_{2}^{ \pm 3}\right\} u_{3, l}, \\
\dot{u}_{3, l} & =-\frac{1}{\Lambda}\left\{2\left(\varpi_{1}+6 \varpi_{3}\right) \mathcal{L}_{3}^{ \pm 1}-30 \varpi_{3} \mathcal{L}_{2}^{ \pm 3}\right\} u_{2, l}-\frac{r}{\Lambda} e^{-2 \lambda}\left\{\left(\left(2-2 r \nu^{\prime}\right)\left(\varpi_{1}+6 \varpi_{3}\right)+r\left(\varpi_{1}^{\prime}+6 \varpi_{3}^{\prime}\right)\right] \mathcal{L}_{2}^{ \pm 1}\right. \\
& \left.-\frac{15}{2}\left[\left(2-2 r \nu^{\prime}\right) \varpi_{3}+r \varpi_{3}^{\prime}\right] \mathcal{L}_{3}^{ \pm 3}\right\} u_{1, l},
\end{aligned}
$$

where $u_{3, l}$ has been redefined as follows:

$$
u_{3, l}=u_{3, l}-\frac{1}{\Lambda} r^{2} e^{-2 \nu}\left[\left(\varpi_{1}+6 \varpi_{3}\right) \mathcal{L}_{2}^{ \pm 1}-\frac{15}{2} \varpi_{3} \mathcal{L}_{3}^{ \pm 3}\right] H_{l} .
$$

Equation (44) of the variable $\xi^{r}$ becomes trivial for barotropic pulsations, where $\Gamma_{1}=\Gamma$. In fact $\xi^{r}$ is no longer an independent dynamical variable as one can argue from equations (22) and (29).

The polar axisymmetric eigenfrequencies are symmetric with respect to the reversal of rotation direction, suggesting that the rotational corrections appear at second order in $\Omega$ [50]. Therefore, for first order slow rotation approximation the axisymmetric modes have the same frequencies as the nonrotating case. However, in equations (47)-(50) implicitly show up some $\mathcal{O}\left(\Omega^{2}\right)$ coupling terms, such as the last term of equations (48)-(49). In fact in accordance with equation (50), the axial velocity $u_{3, l}$ is a dynamical variable at $\mathcal{O}(\Omega)$ perturbative order, while for a nonrotating star it is stationary and its profile can be chosen on the initial time slice. It is evident that this stationary initial condition cannot change the mode frequencies of polar perturbations. Here we use equations (47)-(50) without discarding the $u_{3, l}$ terms in equations (48)-(49), in order to investigate the $\mathcal{O}\left(\Omega^{2}\right)$ corrections of the axial velocity on the polar mode frequencies. However, a complete second order slow rotation approximation is needed for a proper analysis that will take into account all coupling terms.

The system of four coupled equations (47)-(50) has been evolved using second order accurate differencing methods both in time and in space based on the two step Lax-Wendroff algorithm. As a result, the numerical code achieved second order convergence. As initial conditions, for the enthalpy and the three velocity perturbations we set a Gaussian pulse, which excites several nonradial modes. The frequencies of these nonradial modes are then estimated by carrying out a Fast Fourier Transformation (FFT) of the resulting timeseries.

In general, the system of perturbation equations has an infinite series of coupling terms, which depend on $l$. However, we got converging numerical results by keeping a finite number of equations, i.e. up to an $l=l_{\max }$. In fact, starting from a simple configuration, where only a particular $l$ is considered, one can gradually add up extra coupling equations until the results show convergence. An example is shown in table II, where we tabulated the frequencies of the fundamental quasi-radial mode $(F)$ and the first overtone $H_{1}$ for several simulations assuming different $l_{\text {max }}$. The initial perturbed configuration is described by spherical oscillations, i.e. $l_{\max }=0$. By gradually introducing the dipole $l_{\max }=1$ and quadrupole $l_{\max }=2$ oscillations, the quasi-radial mode frequencies have been improved. No further changes have been observed, when $l_{\max }=3$ (or higher) perturbations has been taken into account. Actually, in this case, convergence has been achieved already for $l_{\max }=2$. Since this behavior is shared also by the other nonradial modes, we restricted our study up to $l_{\max }=2$.

The accuracy of our description has been tested against published results from a nonlinear code [32] (SAF from now on) for a sequence of stellar models. The frequencies of the first two quasi-radial $\left(F\right.$ and $\left.H_{1}\right)$ and two $l=2$ nonradial modes $\left({ }^{2} f\right.$ and $\left.{ }^{2} p_{1}\right)$ for some of the B-models are listed in table III Although these stellar models describe very fast rotating stars (see table I), the perturbative results show a good agreement (within the limits of the slow rotation approximation) with those obtained with nonlinear simulations in SAF. In fact, a difference of the order of $7 \%$ appears only after the B6 model. Errors of this order are expected for very fast rotating stars since our results, which are based on the slow rotation approximation (first order in $\Omega$ ), cannot describe the flattening of the star due to the centrifugal force.

An important aspect to emphasize is that our results do not show any splitting of the fundamental quasi-radial mode, as observed in SAF. This result supports the argument in [33], that this effect (splitting) could be an artifact of the Cowling approximation in the non-linear regime. Moreover, we don't observe any significant change in the 


\begin{tabular}{c|c|c|c|c}
\hline \hline Model & $\mathrm{F}(\mathrm{kHz})$ & $H_{1}(\mathrm{kHz})$ & ${ }^{2} f(\mathrm{kHz})$ & ${ }^{2} p_{1}(\mathrm{kHz})$ \\
\hline B0 & $2.706(0 \%)$ & $4.547(0 \%)$ & $1.846(0 \%)$ & $4.100(0 \%)$ \\
B1 & $2.712(2 \%)$ & $4.555(2 \%)$ & $1.895(<1 \%)$ & $4.117(1 \%)$ \\
B3 & $2.735(4 \%)$ & $4.578(4 \%)$ & $1.915(1 \%)$ & $4.124(2 \%)$ \\
B6 & $2.797(6 \%)$ & $4.624(4 \%)$ & $1.944(1 \%)$ & $4.134(7 \%)$ \\
B9 & $2.885(8 \%)$ & $4.686(6 \%)$ & $1.974(8 \%)$ & $4.147(14 \%)$ \\
\hline \hline
\end{tabular}

TABLE III: Frequencies of the first two quasi-radial $\left(F\right.$ and $\left.H_{1}\right)$ and nonradial $\left({ }^{2} f\right.$ and $\left.{ }^{2} p_{1}\right)$ modes, for differentially rotating B-models with $A=12$ and $l_{\max }=2$. The relative difference between the perturbative and nonlinear results [32] is shown in parenthesis

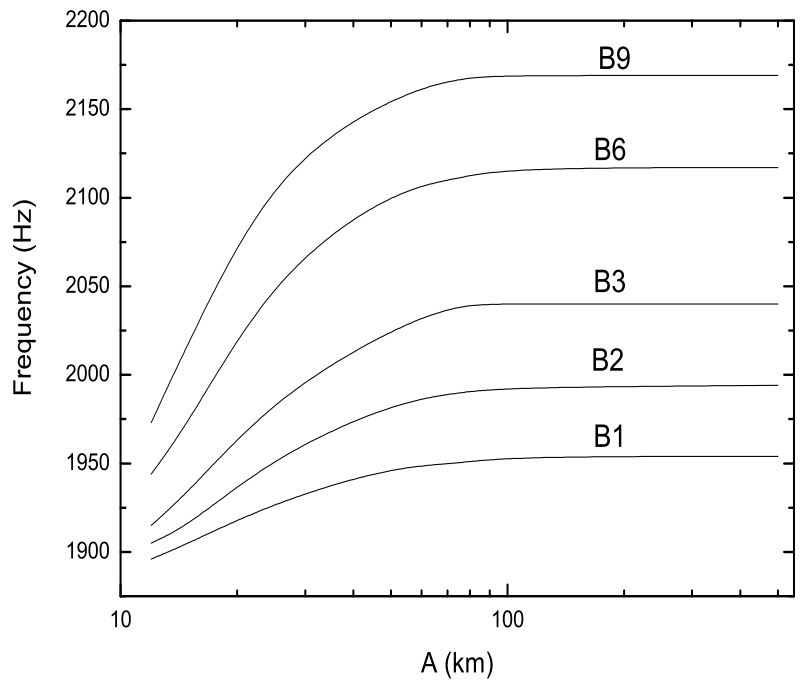

FIG. 5: The frequency of the ${ }^{2} f$ mode as function of the parameter $A$ is plotted for various B-models. For $A=500 \mathrm{~km}$ the star is practically rotates uniformly. The shifting of the mode frequency between the maximum and minimum values of $\mathrm{A}$ varies from $3 \%$ to $10 \%$ depending on the model.

frequency of the ${ }^{2} p_{1}$-mode in constrast to the results in SAF where an overall change of the order of $30 \%$ has been observed.

The degree of differential rotation affects the mode frequencies due to the presence of extra coupling terms in the perturbation equations (40)-(44). This effect can then be studied for several B-stellar models by varying the parameter $A$, from a highly differentially rotating configuration with $A=12 \mathrm{~km}$ to a uniformly rotating star with a very large $A$ e.g. $A=500 \mathrm{~km}$. As shown in figure IV the frequency of the nonradial fundamental ${ }^{2} f$ mode increases as the parameter $A$ increases for the whole sequence of B-models. In particular, the ${ }^{2} f$ frequency already converges to its value for uniform rotation when $A \approx 100 \mathrm{~km}$. In this case, the angular velocity at the equator $\Omega_{e}$ is typically about $2.5 \%$ smaller than $\Omega_{c}$ on the rotational axis. It is worth noticing that the effect of $A$ on the mode frequencies is considerably larger for fast rotating stellar model. As shown in table IV the same behavior has been observed also for the other quasi-radial and nonradial modes. Among these modes, the $F$-mode is the most sensitive to the differential rotation, as it can change up to $25 \%$ for the fastest rotating model B9. Instead, the ${ }^{2} f$ and ${ }^{2} p_{1}$ modes can change up to 10 percent and 3 percent respectively. 


\begin{tabular}{|c|c|c|c|c|c|c|}
\hline Modes & $\mathrm{A}[\mathrm{km}]$ & B0 & B1 & B3 & B6 & B9 \\
\hline \multirow{4}{*}{$\mathrm{F}$} & 12 & 2.706 & 2.702 & 2.735 & 2.797 & 2.885 \\
\hline & 50 & 2.706 & 2.738 & 2.849 & 3.058 & 3.352 \\
\hline & 75 & 2.706 & 2.764 & 2.954 & 3.294 & 3.747 \\
\hline & 100 & 2.706 & 2.771 & 2.960 & 3.313 & 3.777 \\
\hline \multirow{4}{*}{${ }^{2} f$} & 12 & 1.846 & 1.895 & 1.915 & 1.944 & 1.974 \\
\hline & 50 & 1.846 & 1.928 & 1.987 & 2.058 & 2.117 \\
\hline & 75 & 1.846 & 1.947 & 2.038 & 2.111 & 2.169 \\
\hline & 100 & 1.846 & 1.954 & 2.040 & 2.118 & 2.170 \\
\hline \multirow{4}{*}{${ }^{2} p_{1}$} & 12 & 4.100 & 4.117 & 4.124 & 4.134 & 4.147 \\
\hline & 50 & 4.100 & 4.124 & 4.143 & 4.163 & 4.196 \\
\hline & 75 & 4.100 & 4.130 & 4.155 & 4.175 & 4.209 \\
\hline & 100 & 4.100 & 4.134 & 4.157 & 4.176 & 4.210 \\
\hline
\end{tabular}

TABLE IV: Frequencies, in kHz, of the quasi-radial $\mathrm{F}$ mode, and ${ }^{2} f$ and ${ }^{2} p_{1}$ nonradial modes for B-stellar models with different rotational parameter $A$.

\section{CONCLUSIONS AND DISCUSSION}

In this article we derived the general equations describing the perturbations of slowly and differentially rotating neutron stars in the Cowling approximation. The equations have been derived for spherical stars (slow rotation) on which the differential rotation has been described via the $\mathrm{j}$-constant law.

As a first step and test, results have been derived for axisymmetric, barotropic oscillations. In the system of perturbation equations some terms of second order $\mathcal{O}\left(\Omega^{2}\right)$ have been maintained and their effects on the axisymmetric eigenfrequencies has been studied. The comparison of the derived results with those produced by the full non-linear code [32] suggests that this perturbative approach can provide quite accurate estimates of the oscillation frequencies within the limits of the slow rotation approximation. A point to be stressed is that since we keep only terms first order in rotation $\mathcal{O}(\Omega)$ the background stellar model is still spherical, and the compactness of the star $M / R$ remains the same along the sequence of stellar models. This seems to be one of the most important reasons for the deviations observed between our results and those of SAF for the faster rotating members of the B-model sequence. It is expected that the inclusion of second order terms in rotation, which will take into account the flattening of the star, will improve the agreement between the perturbative results with the non-linear ones for the very fast rotating models.

An advantage of this perturbative approach is the numerical simplicity and the ability for an easier physical interpretation of the derived results. In a follow up paper we study the non-axisymmetric oscillations of differentially rotating relativistic stars for various EoS with special emphasis to the hot EoS.

Acknowledgments: We are grateful to Nikolaos Stergioulas, Hajime Sotani and Miltos Vavoulidis for helpful discussions. A.S. is supported by the Pythagoras II grand of the Greek Ministry of Research and Development. A.P. is supported by a "Virgo Ego Scientific Forum" (VESF) and by the EU program ILIAS.

\section{APPENDIX A: HARMONIC COMPONENTS OF THE J-CONSTANT ROTATION LAW}

In this Appendix we present the explicit expression for the terms $\mathcal{I}_{l}^{N}$ and $\mathcal{I}_{l}^{l^{\prime}}$ described in equations (10) and (13). Since the series expansions (6) -(7) for $\omega$ and $\Omega$ have been truncated for $l>3$, and that terms with even $l$ vanish due to the rotation law parity, we show the expressions for harmonic indices $l=1$ and $l=3$. The two nearly "Newtonian terms" $\mathcal{I}_{l}^{N}$ are: 


$$
\begin{aligned}
& \mathcal{I}_{1}^{N}=\frac{3}{2} \frac{A^{2}}{\chi^{2}} \Omega_{c}\left[1-\frac{A^{2}}{\chi \sqrt{A^{2}+\chi^{2}}} \ln \left(\frac{\sqrt{\sqrt{A^{2}+\chi^{2}}+\chi}}{\sqrt{\sqrt{A^{2}+\chi^{2}}-\chi}}\right)\right], \\
& \mathcal{I}_{3}^{N}=\frac{7}{12} \frac{A^{2}}{\chi^{2}} \Omega_{c}\left[1+7.5 \frac{A^{2}}{\chi^{2}}-\frac{6 A^{2}}{\chi \sqrt{A^{2}+\chi^{2}}}\left(1+\frac{5}{4} \frac{A^{2}}{\chi^{2}}\right) \ln \left(\frac{\sqrt{\sqrt{A^{2}+\chi^{2}}+\chi}}{\sqrt{\sqrt{A^{2}+\chi^{2}}-\chi}}\right)\right],
\end{aligned}
$$

The four terms that contribute in the estimation of the "relativistic corrections" $\mathcal{I}_{l}^{R}$ are:

$$
\begin{aligned}
\mathcal{J}_{1}^{1}= & \frac{4}{3} \frac{\pi}{\chi^{4}}\left[2 \chi^{2}-3 A^{2}+\frac{3 A^{4}}{\chi \sqrt{A^{2}+\chi^{2}}} \ln \left(\frac{\sqrt{\sqrt{A^{2}+\chi^{2}}+\chi}}{\sqrt{\sqrt{A^{2}+\chi^{2}}-\chi}}\right)\right], \\
\mathcal{J}_{1}^{3}= & \mathcal{J}_{3}^{1}=-2 \pi \frac{A^{2}}{\chi^{6}}\left[2 \chi^{2}+15 A^{2}-\frac{3 A^{2}\left(4 \chi^{2}+5 A^{2}\right)}{\chi \sqrt{A^{2}+\chi^{2}}} \ln \left(\frac{\sqrt{\sqrt{A^{2}+\chi^{2}}+\chi}}{\sqrt{\sqrt{A^{2}+\chi^{2}}-\chi}}\right)\right], \\
\mathcal{J}_{3}^{3}= & \frac{3}{7} \frac{\pi}{\chi^{8}}\left[-525 A^{6}-490 A^{4} \chi^{2}-56 A^{2} \chi^{4}+16 \chi^{6}+\frac{A^{4}}{\chi \sqrt{A^{2}+\chi^{2}}}\left(525 A^{4}+840 A^{2} \chi^{2}+336 \chi^{4}\right)\right. \\
& \left.\times \ln \left(\frac{\sqrt{\sqrt{A^{2}+\chi^{2}}+\chi}}{\sqrt{\sqrt{A^{2}+\chi^{2}}-\chi}}\right)\right],
\end{aligned}
$$

where we have set $\chi=r e^{-\nu}$.

\section{APPENDIX B: COEFFICIENTS OF THE PERTURBED CONSERVATION EQUATIONS}

In this Appendix we present the coefficients of the perturbation equations (36)-(39),

$$
\begin{aligned}
A_{l m}^{(t)} & =-\dot{H}_{l m}-c_{s}^{2} l(l+1) \frac{e^{2 \nu}}{r^{2}} u_{2, l m}+\left\{\left[\left(\frac{2}{r}-\lambda^{\prime}+2 \nu^{\prime}\right) c_{s}^{2}-\nu^{\prime}\right] u_{1, l m}+c_{s}^{2} u_{1, l m}^{\prime}\right\} e^{2(\nu-\lambda)}, \\
B_{1, l m}^{(t)} & =\left[-\Omega_{1}-6 \Omega_{3}+c_{s}^{2}\left(\varpi_{1}+6 \varpi_{3}\right)\right] H_{l m}, \\
B_{2, l m}^{(t)} & =-\frac{15}{2}\left(c_{s}^{2} \varpi_{3}-\Omega_{3}\right) H_{l m}, \\
A_{l m}^{(r)} & =-\dot{u}_{1, l m}+H_{l m}^{\prime}+\nu^{\prime} c_{s}^{-2}\left[\xi_{l m}+\left(1-\frac{\Gamma_{1}}{\Gamma}\right) H_{l m}\right], \\
B_{1, l m}^{(r)} & =\left\{\left[2\left(\frac{1}{r}-\nu^{\prime}\right) \varpi_{1}-\omega_{1}^{\prime}\right]+6\left[2\left(\frac{1}{r}-\nu^{\prime}\right) \varpi_{3}-\omega_{3}^{\prime}\right]\right\} u_{2, l m} \\
& -\left(\Omega_{1}+6 \Omega_{3}\right) u_{1, l m}, \\
B_{2, l m}^{(r)} & =\frac{15}{2} \Omega_{3} u_{1, l m}-\frac{15}{2}\left[2 \varpi_{3}\left(\frac{1}{r}-\nu^{\prime}\right)-\omega_{3}^{\prime}\right] u_{2, l m}, \\
C_{1, l m}^{(r)} & =\left\{\left[2\left(\frac{1}{r}-\nu^{\prime}\right) \varpi_{1}-\omega_{1}^{\prime}\right]+6\left[2\left(\frac{1}{r}-\nu^{\prime}\right) \varpi_{3}-\omega_{3}^{\prime}\right]\right\} u_{3, l m} \\
C_{2, l m}^{(r)} & =-\frac{15}{2}\left[2 \varpi_{3}\left(\frac{1}{r}-\nu^{\prime}\right)-\omega_{3}^{\prime}\right] u_{3, l m},
\end{aligned}
$$




$$
\begin{aligned}
& a_{1, l m}=-\dot{u}_{2, l m}+H_{l m}-\mathrm{i} m\left(\Omega_{1}+6 \Omega_{3}\right) u_{2, l m}, \\
& a_{2, l m}=2\left(\varpi_{1}+6 \varpi_{3}\right) u_{3, l m}, \\
& a_{3, l m}=\frac{15}{2} \mathrm{i} m \Omega_{3} u_{2, l m} \\
& a_{4, l m}^{(\theta)}=-15\left(\Omega_{3}-2 \omega_{3}\right) u_{3, l m}, \\
& a_{4, l m}^{(\phi)}=-30 \varpi_{3} u_{3, l m}, \\
& b_{1, l m}=-\dot{u}_{3, l m}-\mathrm{i} m\left(\Omega_{1}+6 \Omega_{3}\right) u_{3, l m} \\
& b_{2, l m}=-2\left[\varpi_{1}+6 \varpi_{3}\right] u_{2, l m}, \\
& b_{3, l m}=\frac{15}{2} \mathrm{i} m \Omega_{3} u_{3, l m}, \\
& b_{4, l m}^{(\theta)}=15\left(\Omega_{3}-2 \omega_{3}\right) u_{2, l m}, \\
& b_{4, l m}^{(\phi)}=30 \varpi_{3} u_{2, l m}, \\
& c_{1, l m}^{(\phi)}=c_{s}^{2}\left(\varpi_{1}+6 \varpi_{3}\right)\left(r^{2} e^{-2 \lambda} u_{1, l m}^{\prime}-\Lambda u_{2, l m}\right) \\
& -r e^{-2 \lambda}\left\{\left[2-r \nu^{\prime}-c_{s}^{2}\left(2+r\left(2 \nu^{\prime}-\lambda^{\prime}\right)\right)\right]\left(\varpi_{1}+6 \varpi_{3}\right)+r\left(\varpi_{1}^{\prime}+6 \varpi_{3}^{\prime}\right)\right\} u_{1, l m} \\
& =r^{2} e^{-2 \nu}\left(\varpi_{1}+6 \varpi_{3}\right) \dot{H}_{l m}-r e^{-2 \lambda}\left[\left(2-2 r \nu^{\prime}\right)\left(\varpi_{1}+6 \varpi_{3}\right)+r\left(\varpi_{1}^{\prime}+6 \varpi_{3}^{\prime}\right)\right] u_{1, l m} \text {, } \\
& c_{2, l m}^{(\phi)}=\frac{15}{2} c_{s}^{2} \varpi_{3}\left(\Lambda u_{2, l m}-r^{2} e^{-2 \lambda} u_{1, l m}^{\prime}\right) \\
& +\frac{15}{2} r e^{-2 \lambda}\left\{\left[2-r \nu^{\prime}-c_{s}^{2}\left(2+r\left(2 \nu^{\prime}-\lambda^{\prime}\right)\right)\right] \varpi_{3}+r \varpi_{3}^{\prime}\right\} u_{1, l m} \\
& =-\frac{15}{2} r^{2} e^{-2 \nu} \varpi_{3} \dot{H}_{l m}+\frac{15}{2} r e^{-2 \lambda}\left[\left(2-2 r \nu^{\prime}\right) \varpi_{3}+r \varpi_{3}^{\prime}\right] u_{1, l m},
\end{aligned}
$$

where we have used the definitions,

$$
\begin{aligned}
& \varpi_{1}(r) \equiv \Omega_{1}(r)-\omega_{1}(r), \\
& \varpi_{3}(r) \equiv \Omega_{3}(r)-\omega_{3}(r) .
\end{aligned}
$$

\section{APPENDIX C: ANGULAR INTEGRATION}

Here, we define the linear operators $\mathcal{L}_{i}^{ \pm j}$ with $i, j \in \mathbb{N}$, which come out from the angular integration of equations (36)(39). This procedure relies on the orthogonality properties of the spherical harmonics, on the following angular integrals:

$$
\begin{aligned}
\int_{S^{2}} d \tilde{\Omega}( & \left.\frac{\partial Y_{l^{\prime} m^{\prime}}^{*}}{\partial \theta} \frac{\partial Y_{l m}}{\partial \theta}+\frac{1}{\sin ^{2} \theta} \frac{\partial Y_{l^{\prime} m^{\prime}}^{*}}{\partial \phi} \frac{\partial Y_{l m}}{\partial \phi}\right)=l(l+1) \delta_{l l^{\prime}} \delta_{m m^{\prime}} \\
\int_{S^{2}} d \tilde{\Omega}\left(Y_{l^{\prime} m^{\prime}}^{*} \frac{\partial Y_{l m}}{\partial \theta}+\frac{\partial Y_{l^{\prime} m^{\prime}}^{*}}{\partial \theta} Y_{l m}\right) \frac{\cos \theta}{\sin \theta} & =\delta_{l l^{\prime}} \delta_{m m^{\prime}}
\end{aligned}
$$

and the two relations:

$$
\begin{aligned}
\cos \theta Y_{l m} & =Q_{l+1 m} Y_{l+1 m}+Q_{l m} Y_{l-1 m} \\
\sin \theta \partial_{\theta} Y_{l m} & =Q_{l+1 m} l Y_{l+1 m}-Q_{l m}(l+1) Y_{l-1 m}
\end{aligned}
$$


where $Q_{l m}$ is defined as follows:

$$
Q_{l m}=\sqrt{\frac{(l-m)(l+m)}{(2 l-1)(2 l+1)}} .
$$

The operators $\mathcal{L}_{j}^{ \pm 1}$ which introduce couplings of a perturbation term/expression with harmonic index $l$ with the term/expression having harmonic indices $l \pm 1$ are given by the following relations:

$$
\begin{aligned}
\mathcal{L}_{1}^{ \pm 1} \mathcal{A} & \equiv \sum_{l^{\prime} m^{\prime}} \mathcal{A}_{l^{\prime} m^{\prime}} \int_{S^{2}} d \tilde{\Omega} Y_{l m}^{*} \sin \theta \partial_{\theta} Y_{l^{\prime} m^{\prime}}=(l-1) Q_{l m} \mathcal{A}_{l-1 m}-(l+2) Q_{l+1 m} \mathcal{A}_{l+1 m} \\
\mathcal{L}_{2}^{ \pm 1} \mathcal{A} & \equiv \sum_{l^{\prime} m^{\prime}} \mathcal{A}_{l^{\prime} m^{\prime}} \int_{S^{2}} d \tilde{\Omega} \partial_{\theta} Y_{l m}^{*} \sin \theta Y_{l^{\prime} m^{\prime}}=-(l+1) Q_{l m} \mathcal{A}_{l-1 m}+l Q_{l+1 m} \mathcal{A}_{l+1 m} \\
\mathcal{L}_{3}^{ \pm 1} \mathcal{A} & \equiv \sum_{l^{\prime} m^{\prime}} \mathcal{A}_{l^{\prime} m^{\prime}} \int_{S^{2}} d \tilde{\Omega}\left(\partial_{\theta} Y_{l m}^{*} \partial_{\theta} Y_{l^{\prime} m^{\prime}}+\frac{1}{\sin ^{2} \theta} \partial_{\phi} Y_{l m}^{*} \partial_{\phi} Y_{l^{\prime} m^{\prime}}\right) \cos \theta \\
& =(l-1)(l+1) Q_{l m} \mathcal{A}_{l-1 m}+l(l+2) Q_{l+1 m} \mathcal{A}_{l+1 m} \\
\mathcal{L}_{4}^{ \pm 1} \mathcal{A} & \equiv \sum_{l^{\prime} m^{\prime}} \mathcal{A}_{l^{\prime} m^{\prime}} \int_{S^{2}} d \tilde{\Omega} Y_{l m}^{*} \cos \theta Y_{l^{\prime} m^{\prime}}=Q_{l m} \mathcal{A}_{l-1 m}+Q_{l+1 m} \mathcal{A}_{l+1 m} \\
\mathcal{L}_{5}^{ \pm 1} \mathcal{A} & \equiv \sum_{l^{\prime} m^{\prime}} \mathcal{A}_{l^{\prime} m^{\prime}} \int_{S^{2}} d \tilde{\Omega}\left(\partial_{\theta} Y_{l m}^{*} \partial_{\phi} Y_{l^{\prime} m^{\prime}}-\partial_{\phi} Y_{l m}^{*} \partial_{\theta} Y_{l^{\prime} m^{\prime}}\right) \sin \theta=\mathrm{i} m\left(\mathcal{L}_{1}^{ \pm 1}+\mathcal{L}_{2}^{ \pm 1}\right)
\end{aligned}
$$

Notice that the operator $\mathcal{L}_{4}^{ \pm 1}$ can be defined in terms of the $\mathcal{L}_{1}^{ \pm 1}$ and $\mathcal{L}_{2}^{ \pm 1}$ operators as follows:

$$
\mathcal{L}_{4}^{ \pm 1}=-\frac{1}{2}\left(\mathcal{L}_{1}^{ \pm 1}+\mathcal{L}_{2}^{ \pm 1}\right)
$$

In a similar way the operators $\mathcal{L}_{j}^{ \pm 2}$ that introduce couplings of a perturbation expression with harmonic index $l$ with term/expression with harmonic indices $l \pm 2$ are:

$$
\begin{aligned}
\mathcal{L}_{1}^{ \pm 2} \mathcal{A} \equiv & \sum_{l^{\prime} m^{\prime}} \mathcal{A}_{l^{\prime} m^{\prime}} \int_{S^{2}} d \tilde{\Omega} Y_{l m}^{*} \sin ^{2} \theta Y_{l^{\prime} m^{\prime}}=-Q_{l-1 m} Q_{l m} \mathcal{A}_{l-2 m}+\left(1-Q_{l m}^{2}-Q_{l+1 m}^{2}\right) \mathcal{A}_{l m} \\
& -Q_{l m} Q_{l+1 m} \mathcal{A}_{l+2 m} \\
\mathcal{L}_{2}^{ \pm 2} \mathcal{A} \equiv & \sum_{l^{\prime} m^{\prime}} \mathcal{A}_{l^{\prime} m^{\prime}} \int_{S^{2}} d \tilde{\Omega} \partial_{\theta} Y_{l m}^{*} \sin \theta \cos \theta Y_{l^{\prime} m^{\prime}}=-(l+1) Q_{l-1 m} Q_{l m} \mathcal{A}_{l-2 m}+\left[l Q_{l+1 m}^{2}-(l+1) Q_{l m}^{2}\right] \mathcal{A}_{l m} \\
& +l Q_{l+1 m} Q_{l+2 m} \mathcal{A}_{l+2 m}, \\
\mathcal{L}_{3}^{ \pm 2} \mathcal{A} \equiv & \sum_{l^{\prime} m^{\prime}} \mathcal{A}_{l^{\prime} m^{\prime}} \int_{S^{2}} d \tilde{\Omega} Y_{l m}^{*} \sin \theta \cos \theta \partial_{\theta} Y_{l^{\prime} m^{\prime}}=(l-2) Q_{l-1 m} Q_{l m} \mathcal{A}_{l-2 m}+\left[l Q_{l+1 m}^{2}-(l+1) Q_{l m}^{2}\right] \mathcal{A}_{l m} \\
& -(l+3) Q_{l+1 m} Q_{l+2 m} \mathcal{A}_{l+2 m}, \\
\mathcal{L}_{4}^{ \pm 2} \mathcal{A} \equiv & \sum_{l^{\prime} m^{\prime}} \mathcal{A}_{l^{\prime} m^{\prime}} \int_{S^{2}} d \tilde{\Omega}\left(\partial_{\theta} Y_{l m}^{*} \partial_{\theta} Y_{l^{\prime} m^{\prime}}+\frac{1}{\sin ^{2} \theta} \partial_{\phi} Y_{l m}^{*} \partial_{\phi} Y_{l^{\prime} m^{\prime}}\right) \sin ^{2} \theta=-(l-2)(l+1) Q_{l-1 m} Q_{l m} \mathcal{A}_{l-2 m} \\
& +\left[l(l+1)-(l+1)(l-2) Q_{l m}^{2}-l(l+3) Q_{l+1 m}^{2}\right] \mathcal{A}_{l m}-l(l+3) Q_{l+1 m} Q_{l+2 m} \mathcal{A}_{l+2 m}
\end{aligned}
$$

Finally, the operators $\mathcal{L}_{j}^{ \pm 3}$ that introduce couplings of a perturbation expression with harmonic index $l$ with terms/expressions with harmonic indices $l \pm 3$ are: 


$$
\begin{aligned}
\mathcal{L}_{1}^{ \pm 3} \mathcal{A} \equiv & \sum_{l^{\prime} m^{\prime}} \mathcal{A}_{l^{\prime} m^{\prime}} \int_{S^{2}} d \tilde{\Omega} Y_{l m}^{*} \sin ^{3} \theta \partial_{\theta} Y_{l^{\prime} m^{\prime}}=-(l-3) Q_{l-2 m} Q_{l-1 m} Q_{l m} \mathcal{A}_{l-3 m}+(l+4) Q_{l+1 m} Q_{l+2 m} Q_{l+3 m} \mathcal{A}_{l+3 m} \\
& +Q_{l m}\left[l Q_{l-1 m}^{2}+(l-1)\left(1-Q_{l m}^{2}-Q_{l+1 m}^{2}\right)\right] \mathcal{A}_{l-1 m} \\
& -Q_{l+1 m}\left[(l+1) Q_{l+2 m}^{2}+(l+2)\left(1-Q_{l m}^{2}-Q_{l+1 m}^{2}\right)\right] \mathcal{A}_{l+1 m}, \\
\mathcal{L}_{2}^{ \pm 3} \mathcal{A} \equiv & \sum_{l^{\prime} m^{\prime}} \mathcal{A}_{l^{\prime} m^{\prime}} \int_{S^{2}} d \tilde{\Omega} \partial_{\theta} Y_{l m}^{*} \cos \theta \sin ^{2} \theta \partial_{\theta} Y_{l^{\prime} m^{\prime}}=-(l-3)(l+1) Q_{l-2 m} Q_{l-1 m} Q_{l m} \mathcal{A}_{l-3 m} \\
& +Q_{l m}\left[l(l+1) Q_{l-1 m}^{2}-(l-1)(l+1) Q_{l m}^{2}+l(l-1) Q_{l+1 m}^{2}\right] \mathcal{A}_{l-1 m} \\
& +Q_{l+1 m}\left[(l+1)(l+2) Q_{l m}^{2}-l(l+2) Q_{l+1 m}^{2}+l(l+1) Q_{l+2 m}^{2}\right] \mathcal{A}_{l+1 m} \\
& -l(l+4) Q_{l+1 m} Q_{l+2 m} Q_{l+3 m} \mathcal{A}_{l+3 m}, \\
\mathcal{L}_{3}^{ \pm 3} \mathcal{A} \equiv & \sum_{l^{\prime} m^{\prime}} \mathcal{A}_{l^{\prime} m^{\prime}} \int_{S^{2}} d \tilde{\Omega} \partial_{\theta} Y_{l m}^{*} \sin ^{3} \theta Y_{l^{\prime} m^{\prime}}=(l+1) Q_{l-2 m} Q_{l-1 m} Q_{l m} \mathcal{A}_{l-3 m} \\
& -Q_{l m}\left[(l+1)+l Q_{l+1 m}^{2}-(l+1)\left(Q_{l-1 m}^{2}+Q_{l m}^{2}\right)\right] \mathcal{A}_{l-1 m} \\
& +Q_{l+1 m}\left[l+(l+1) Q_{l m}^{2}-l\left(Q_{l+1 m}^{2}+Q_{l+2 m}^{2}\right)\right] \mathcal{A}_{l+1 m} \\
& -l Q_{l+1 m} Q_{l+2 m} Q_{l+3 m} \mathcal{A}_{l+3 m} .
\end{aligned}
$$

\section{APPENDIX D: ROTATION LAW IN ISOTROPIC COORDINATES}

In this Appendix, we describe the j-constant rotation law in isotropic coordinates, and the appropriate transformation in Schwarzshild coordinates. In isotropic coordinates the axially symmetric spacetime for a slowly rotating body can be described by the following metric:

$$
d s^{2}=-e^{2 \alpha} d t^{2}+e^{2 \beta}\left[d \hat{r}^{2}+\hat{r}^{2}\left(d \theta^{2}+\sin ^{2} \theta d \phi^{2}\right)\right]-2 e^{2 \beta} \omega \hat{r}^{2} \sin ^{2} \theta d t d \phi,
$$

where $\alpha$ and $\beta$ are functions of the isotropic coordinate $\hat{r}$. Equation (D1) has been derived by the slow rotating metric (1) in Schwarschild coordinate via the following relations:

$$
\begin{aligned}
\frac{d \hat{r}}{d r} & =\frac{\hat{r}}{r}\left(1-\frac{2 M}{r}\right)^{-\frac{1}{2}}, \\
e^{\beta} & =\frac{r}{\hat{r}}
\end{aligned}
$$

where $M=M(r)$ is the mass function in Schwarzshild coordinates. At the stellar surface the metric functions must smoothly join the Schwarzshild solution, this leads to the following matching conditions in isotropic coordinates

$$
\begin{aligned}
e^{\alpha} & =\left(1-\frac{M}{2 \hat{r}}\right)\left(1+\frac{M}{2 \hat{r}}\right)^{-1}, \\
e^{\beta} & =\left(1+\frac{M}{2 \hat{r}}\right)^{2}
\end{aligned}
$$

The stellar radius $\hat{R}$, in isotropic coordinates, is given in terms of the stellar mass $M$ and the radius $R$ in Schwarzshild coordinates by the following relation:

$$
\hat{R}=\frac{1}{2}\left(R-M+\sqrt{R^{2}-2 M R}\right) .
$$

Finally, the relativistic $\mathrm{j}$-constant rotation law can be determined via the relation:

$$
u^{t} u_{\phi}=\hat{A}^{2}\left(\Omega_{c}-\Omega\right),
$$


which leads to the following expression of the angular velocity profile:

$$
\Omega(\hat{r}, \theta)=\frac{\hat{A}^{2} \Omega_{c}+e^{2(\beta-\alpha)} \hat{r}^{2} \sin ^{2} \theta \omega(\hat{r}, \theta)}{\hat{A}^{2}+e^{2(\beta-\alpha)} \hat{r}^{2} \sin ^{2} \theta},
$$

where $\hat{A}$ is a parameter that describes the degree of differential rotation in isotropic coordinates. By defining a new function

$$
\hat{x}=e^{\beta-\alpha} \frac{\hat{r}}{\hat{A}} \sin \theta,
$$

we can rewrite equation (D8) in a more compact form i.e.

$$
\frac{\Omega(\hat{r}, \theta)}{\Omega_{c}}=\frac{1+\hat{x}^{2} \omega(\hat{r}, \theta) / \Omega_{c}}{1+\hat{x}^{2}} .
$$

It is worth noticing that equation (5) can be written in the same form of equation (D10) if the function $\hat{x}$ is replaced by

$$
x=e^{-\nu} \frac{r}{A} \sin \theta
$$

Equations (D8) and (5) are obviously related by the coordinate transformation between isotropic and Schwarzshild coordinates. A typical choice for $\hat{A}$ is to set its value equal to the stellar radius $\hat{R}$, which, as we show later, corresponds to $\Omega_{e} \sim \Omega_{c} / 3$. This relation between the angular velocity at the equator and the rotation axis seems to be in good agreement with the rotation patters estimated in the remnants of hypermassive binary mergers [4]. The matching conditions (D4) and (D5) lead to the following expression for $\hat{x}_{e}$ at the equator:

$$
\hat{x}_{e}=\left(1+\frac{M}{2 \hat{R}}\right)^{3}\left(1-\frac{M}{2 \hat{R}}\right)^{-1} \frac{\hat{R}}{\hat{A}} .
$$

A coordinate transformation leads to the following expression for $x_{e}$ in Schwarzshild coordinates:

$$
x_{e}=\left(1-\frac{2 M}{R}\right)^{-1 / 2} \frac{R}{\hat{A}} .
$$

From equations (D10)-(D13), it is worth noticing that the ratio $\Omega_{e} / \Omega_{c}$ depends practically on the compactness of the star. In order to set up an equilibrium configuration in Schwarzshild coordinates, which has the same value of $\Omega_{e} / \Omega_{c}$ as the one used in non-linear calculations in isotropic coordinates, the natural choice is $A=\hat{A}$ as it comes out from the equations (D13) and (D11). For example, for a polytropic stellar model with mass $M=1.4 M_{\odot}$ and radius $R=14.151 \mathrm{~km}$, equation (D6) gives $\hat{R}=12 \mathrm{~km}$ and leads to $\hat{x}_{e}=1.4$. By neglecting the corrections given by the metric function $\omega$ in equations $\left(\right.$ (D8) and (5), the choice $A=\hat{R}$ leads to $x_{e}=\hat{x_{e}}$ and $\Omega_{e}=0.33 \Omega_{c}$ in both coordinate systems.

[1] H. Dimmelmeier, J. A. Font, and E. Müller, A\&A 393, 523 (2002), astro-ph/0204289.

[2] C. D. Ott, A. Burrows, E. Livne, and R. Walder, ApJ 600, 834 (2004), astro-ph/0307472.

[3] F. A. Rasio and S. L. Shapiro, ApJ 432, 242 (1994), astro-ph/9401027.

[4] M. Shibata and K. Uryu, Phys. Rev. D61, 064001 (2000), gr-qc/9911058.

[5] L. Rezzolla, F. K. Lamb, and S. L. Shapiro, ApJ 531, L139 (2000), astro-ph/9911188.

[6] Y. Levin and G. Ushomirsky, MNRAS 324, 917 (2001), astro-ph/0006028.

[7] N. Stergioulas and J. A. Font, Physical Review Letters 86, 1148 (2001), gr-qc/0007086.

[8] P. M. Sá, Phys. Rev. D 69, 084001 (2004), gr-qc/0302115.

[9] S. L. Shapiro, ApJ 544, 397 (2000), astro-ph/0010493.

[10] J. N. Cook, S. L. Shapiro, and B. C. Stephens, ApJ 599, 1272 (2003), astro-ph/0310304.

[11] D. J. Hegyi, ApJ 217, 244 (1977).

[12] Y. T. Liu and S. L. Shapiro, Physical Review D (Particles, Fields, Gravitation, and Cosmology) 69, 044009 (pages 27) (2004), URL http://link.aps.org/abstract/PRD/v69/e044009.

[13] B. F. Schutz, Jr., ApJS 24, 319 (1972). 
[14] B. F. Schutz, Jr., ApJS 24, 343 (1972).

[15] F. H. Seguin, ApJ 179, 289 (1973).

[16] F. H. Seguin, ApJ 197, 745 (1975).

[17] C. J. Hansen, J. P. Cox, and H. M. van Horn, ApJ 217, 151 (1977).

[18] S. Karino, S. Yoshida, and Y. Eriguchi, Phys. Rev. D 64, 024003 (2001), astro-ph/0012430.

[19] M. A. Abramowicz, L. Rezzolla, and S. Yoshida, Class. Quant. Grav. 19, 191 (2000), gr-qc/0112042.

[20] L. Rezzolla and S. Yoshida, Class. Quant. Grav. 18, L87 (2001), gr-qc/0107027.

[21] S. Yoshida, L. Rezzolla, S. Karino, and Y. Eriguchi, ApJ 568, L41 (2002), gr-qc/0112017.

[22] M. Shibata, S. Karino, and Y. Eriguchi, MNRAS 334, L27 (2002), gr-qc/0206002.

[23] M. Shibata and Y.-I. Sekiguchi, Phys. Rev. D 71, 024014 (2005), astro-ph/0412243.

[24] J. M. Centrella, K. C. B. New, L. L. Lowe, and J. D. Brown, ApJ 550, L193 (2001), astro-ph/0010574.

[25] M. Saijo, T. W. Baumgarte, and S. L. Shapiro, ApJ 595, 352 (2003), astro-ph/0302436.

[26] S. Ou and J. E. Tohline, ApJ 651, 1068 (2006), astro-ph/0604099.

[27] A. L. Watts, N. Andersson, and D. I. Jones, ApJ 618, L37 (2005), astro-ph/0309554.

[28] M. Saijo and S. Yoshida, MNRAS 368, 1429 (2006), astro-ph/0505543.

[29] Z. B. Etienne, Y. T. Liu, and S. L. Shapiro, Phys. Rev. D74, 044030 (2006), astro-ph/0609634.

[30] M. D. Duez, Y. T. Liu, S. L. Shapiro, and M. Shibata, Phys. Rev. D73, 104015 (2006), astro-ph/0605331.

[31] B. C. Stephens, M. D. Duez, Y. T. Liu, S. L. Shapiro, and M. Shibata (2006), gr-qc/0610103.

[32] N. Stergioulas, T. A. Apostolatos, and J. A. Font, MNRAS 352, 1089 (2004), astro-ph/0312648.

[33] H. Dimmelmeier, N. Stergioulas, and J. A. Font, Mon. Not. Roy. Astron. Soc. 368, 1609 (2006), astro-ph/0511394.

[34] Y. Kojima, Phys. Rev. D46, 4289 (1992).

[35] K. H. Lockitch and J. L. Friedman, ApJ 521, 764 (1999), gr-qc/9812019.

[36] J. Ruoff and K. D. Kokkotas, MNRAS 328, 678 (2001), gr-qc/0101105.

[37] A. Stavridis and K. D. Kokkotas, International Journal of Modern Physics D 14, 543 (2005), gr-qc/0411019.

[38] N. Stergioulas, Living Rev. Rel. 6, 3 (2003), gr-qc/0302034.

[39] H. Komatsu, Y. Eriguchi, and I. Hachisu, MNRAS 237, 355 (1989).

[40] H. Komatsu, Y. Eriguchi, and I. Hachisu, MNRAS 239, 153 (1989).

[41] A. Passamonti, M. Bruni, L. Gualtieri, A. Nagar, and C. F. Sopuerta, Phys. Rev. D73, 084010 (2006), gr-qc/0601001.

[42] J. B. Hartle, ApJ 150, 1005 (1967).

[43] J. B. Hartle, ApJ 161, 111 (1970).

[44] N. Stergioulas and J. L. Friedman, ApJ 444, 306 (1995), astro-ph/9411032.

[45] J. Ruoff, A. Stavridis, and K. D. Kokkotas, MNRAS 332, 676 (2002), gr-qc/0109065.

[46] S. Yoshida and Y. Kojima, MNRAS 289, 117 (1997), gr-qc/9705081.

[47] J. R. Ipser and R. H. Price, Phys. Rev. D43, 1768 (1991).

[48] K. H. Lockitch, N. Andersson, and J. L. Friedman, Phys. Rev. D63, 024019 (2001), gr-qc/0008019.

[49] A. Passamonti, A. Stavridis, and K. D. Kokkotas, in preparation (2007).

[50] J. B. Hartle, K. S. Thorne, and S. M. Chitre, ApJ 176, 177 (1972). 